Annales UMCS

Informatica

Lublin-Polonia

Sectio AI

http://www.annales.umcs.lublin.pl/

\title{
Numerical schemes for a system of one-dimensional hyperbolic equations
}

\author{
Krzysztof Murawski \\ Institute of Physics, Maria Curie Sklodowska University, \\ pl. M. Curie-Sktodowskiej 1, 20-031 Lublin, Poland
}

\begin{abstract}
In this paper modern numerical methods for one-dimensional hyperbolic equations are reviewed. Several results of advanced numerical simulations for complex systems are presented. These results prove that the numerical codes, based on the Godunov-type methods cope very well with all numerically induced problems.
\end{abstract}

\section{Introduction}

The simplest physical phenomena are very often described by complicated mathematical equations, which cannot be solved analytically and require numerical treatment. The basic idea of computer experiments is to simulate the physical behavior of complicated natural systems by solving an appropriate set of mathematical equations that are built on the basis of a physical model. A typical way for computer simulation is to develop a mathematical model, perhaps in a series of differential or integral equations and then to transform them to a discrete form that can be numerically treated. In this way, numerical simulations attempt to initiate the dynamic behavior of a system and to predict or calculate subsequent events.

Numerical simulations have emerged as a new branch in physics complementing both experiments and theory. A simulation can sometimes replace a physical 
experiment, although most often a simulation and an experiment are complementary. Results of scientific experiments are often explained by simulations, and simulations are often calibrated by experiments. The experiments provide input for the simulations which are viewed as experimenting with theoretical models. The feedback of numerical results into theoretical modelling and the continuous interaction with laboratory experiments and analytical theory make computing an indispensable tool for science. Therefore, the increase in computing power in both speed and storage has given computational physics its significance. Improved computer capacity and the solution algorithms themselves, have a large effect on the quality of solutions obtained.

Numerical simulations can be used to study the dynamics of complex physical systems. Although the variety of complex flows that computational fluid dynamics can analyse continues to increase, the solutions to much more complex flows are desired. A numerical model can be used to interpret measurements and observations, extend existing analytical models into new parameter regimes and quantitatively test existing theories. That can be done by comparing model predictions to experimental data.

A goal of this paper is to present modern numerical schemes for solving a set of hyperbolic partial differential equations. This paper is organized as follows. A mathematical theory for the linear system of one-dimensional equations is introduced in the following section. Section 3 presents an extension of this theory for a system of nonlinear equations. The shock tube problem is discussed in Sect. 4. Rankine-Hugoniot jump conditions which describe a shock are presented in Sect. 5. The Riemann problem for the Euler equations is shown in Sect. 6. Some deficiencies of Godunov-type schemes are discussed in Sect. 7. Numerical simulations of random waves are described in Sect. 8. This paper ends with conclusions.

\section{Linear system of one-dimensional equations}

Consider the linear system of equations

$$
\mathbf{u}_{, t}+\mathbf{A} \mathbf{u}_{, x}=0
$$

with the initial condition

$$
\mathbf{u}(x, t=0)=\mathbf{u}_{0}(x) .
$$

Now, both $\mathbf{u}$ and $\mathbf{A}$ are matrices such as $\mathbf{u}: R \times R \rightarrow R^{q}$ and $\mathbf{A} \in R^{q \times q}$. The above system is called hyperbolic if a constant matrix $\mathbf{A}$ is diagonalizable with real eigenvalues and the corresponding set of right eigenvectors is complete (c.f. $[\mathbf{1}])$. If these eigenvalues are distinct for all $\mathbf{u}(x, t)$ the system is called strictly 
hyperbolic. That allows us to decompose the matrix $\mathbf{A}$ in the following way:

$$
\mathbf{A}=\mathbf{R} \mathbf{\Lambda} \mathbf{R}^{-1}
$$

where:

$$
\mathbf{R}=\left(\mathbf{r}^{1}\left|, \mathbf{r}^{2}\right|, \cdots, \mid \mathbf{r}^{q}\right)
$$

is the matrix of right eigenvectors and

$$
\boldsymbol{\Lambda}=\operatorname{diag}\left(\lambda^{1}, \lambda^{2}, \cdots, \lambda^{q}\right)
$$

is a diagonal matrix of eigenvalues.

From Eq. (2) we get

$$
\mathbf{A R}=\mathbf{R} \boldsymbol{\Lambda} .
$$

Hence

$$
\mathbf{A r}^{m}=\lambda^{m} \mathbf{r}^{m}, \quad m=1,2, \cdots, q .
$$

From this equation we can obtain the eigenvalues as

$$
\operatorname{det}\left(\mathbf{A}-\lambda^{m} \mathbf{I}\right)=0,
$$

where $\mathbf{I}$ is the unit matrix.

The matrix $\mathbf{A}$ can be decomposed based on the sign of each eigenvalue $\lambda^{m}$

$$
\mathbf{A}=\mathbf{A}^{+}+\mathbf{A}^{-}, \quad \mathbf{A}^{ \pm}=\mathbf{R} \mathbf{\Lambda}^{ \pm} \mathbf{R}^{-1} .
$$

Here $\boldsymbol{\Lambda}^{+}\left(\boldsymbol{\Lambda}^{-}\right)$consists of the positive (negative) parts of each $\lambda^{m}$ only, $i$. $e$.

$$
\boldsymbol{\Lambda}^{ \pm}=\operatorname{diag}\left(\lambda^{ \pm}\right), \quad \lambda^{+}=\max \left(\lambda^{m}, 0\right), \quad \lambda^{-}=\min \left(\lambda^{m}, 0\right) .
$$

\subsection{Characteristic variables.}

Equation (1) can be solved with the use of characteristic variables

$$
\mathbf{v}(x, t)=\mathbf{R}^{-1} \mathbf{u}(x, t) .
$$

From Eq. (1) we obtain then

$$
\mathbf{v}_{, t}+\Lambda \mathbf{v}_{, x}=0 .
$$

As $\boldsymbol{\Lambda}$ is diagonal the above matrix equation contains $q$ decoupled scalar equations which are solved by

$$
v^{m}(x, t)=v^{m}\left(x-\lambda^{m} t, 0\right), \quad m=1,2, \cdots, q .
$$

From Eq. (5) we get

$$
\mathbf{u}(x, t)=\mathbf{R} \mathbf{v}(x, t)
$$

which is equivalent to

$$
\mathbf{u}(x, t)=\sum_{m=1}^{q} v^{m}(x, t) \mathbf{r}^{m}=\sum_{m=1}^{q} v^{m}\left(x-\lambda^{m} t, 0\right) \mathbf{r}^{m} .
$$


As a consequence of the fact that $\mathbf{u}(x, t)$ depends only on the initial data $v^{m}\left(x-\lambda^{m} t, 0\right)$ at the $q$ points $x-\lambda^{m} t$. The curves $x=x_{0}+\lambda^{m} t$ are called p-characteristics. When $\mathbf{A}$ is a constant matrix, p-characteristics are straight lines.

\subsection{Riemann problem for the linear equations.}

The characteristic variables are very useful for solving the Riemann problem

$$
\mathbf{u}(x, t=0)= \begin{cases}\mathbf{u}_{l} & \text { for } x<0 \\ \mathbf{u}_{r} & \text { for } x>0\end{cases}
$$

with

$$
\mathbf{u}_{l}=\sum_{m=1}^{q} v_{l}^{m} \mathbf{r}^{m}, \quad \mathbf{u}_{r}=\sum_{m=1}^{q} v_{r}^{m} \mathbf{r}^{m}
$$

such that

$$
v^{m}(x, t=0)= \begin{cases}v_{l}^{m} & \text { for } x<0 \\ v_{r}^{m} & \text { for } x>0\end{cases}
$$

This implies

$$
v^{m}(x, t)= \begin{cases}v_{l}^{m} & \text { for } x-\lambda^{m} t<0, \\ v_{r}^{m} & \text { for } x-\lambda^{m} t>0 .\end{cases}
$$

If we design $M(x, t)$ to be the maximum of $m$ for which $x-\lambda^{m} t>0$, then

$$
\mathbf{u}(x, t)=\sum_{m=1}^{M(x, t)} v_{r}^{m} \mathbf{r}^{m}+\sum_{m=M+1}^{q} v_{l}^{m} \mathbf{r}^{m} .
$$

This equation can alternatively be rewritten as follows:

$$
\mathbf{u}(x, t)=\mathbf{u}_{l}+\sum_{\lambda^{m}<x / t}\left(v_{r}^{m}-v_{l}^{m}\right) \mathbf{r}^{m}=\mathbf{u}_{r}-\sum_{\lambda^{m}>x / t}\left(v_{r}^{m}-v_{l}^{m}\right) \mathbf{r}^{m} .
$$

This solution consists of $q$ waves, which are discontinuities propagating at the characteristic velocities, $\lambda^{m}, m=1,2, \cdots, q$, of the system.

It is noteworthy that the solution of the Riemann problem is self-similar, $i$. e. $\mathbf{u}(x, t)=\mathbf{u}(\alpha x, \alpha t)$ for $\alpha>0$.

\subsection{The wave propagation method.}

We present the wave propagation method that was developed by LeVeque $[2,3,4]$ for a linear hyperbolic system of equations of Eq. (1). Then, the difference $\Delta \mathbf{u}_{i}$, evaluated at the spatial point $x_{i}$, can be written as

$$
\Delta \mathbf{u}_{i} \equiv \mathbf{u}_{i}-\mathbf{u}_{i-1}=\sum_{m=1}^{q} \alpha_{i}^{m} \mathbf{r}_{i}^{m} \equiv \sum_{m=1}^{q} \mathbf{W}_{i}^{m},
$$


where $\mathbf{W}_{i}^{m}$ is the wave, $\lambda_{i}^{m}$ is its speed and $\alpha_{i}^{m}$ is the scalar coefficient which measures a strength of the wave $m$ such that

$$
\alpha_{i}=\mathbf{R}^{-1} \Delta \mathbf{u}_{i} .
$$

Let $\mathbf{u}_{i}^{0}$ denote the value at the interface between $\mathbf{u}_{i-1}$ and $\mathbf{u}_{i}$. Then, with the use of (6) we can write

$$
\mathbf{u}_{i}^{0}-\mathbf{u}_{i-1}=\sum_{\lambda_{i}^{m}<0} \mathbf{W}_{i}^{m}, \quad \mathbf{u}_{i}-\mathbf{u}_{i}^{0}=\sum_{\lambda_{i}^{m}>0} \mathbf{W}_{i}^{m} .
$$

The flux at the interface can be expressed twofold:

$$
\begin{aligned}
\mathbf{f}\left(\mathbf{u}_{i}^{0}\right) & =\mathbf{A} \mathbf{u}_{i}^{0}=\mathbf{A} \mathbf{u}_{i-1}+\sum_{\lambda_{i}^{m}<0} \lambda_{i}^{m} \mathbf{W}_{i}^{m} \equiv \mathbf{A} \mathbf{u}_{i-1}+\mathbf{A}^{-} \Delta \mathbf{u}_{i}, \\
\mathbf{f}\left(\mathbf{u}_{i}^{0}\right) & =\mathbf{A} \mathbf{u}_{i}^{0}=\mathbf{A} \mathbf{u}_{i}-\sum_{\lambda_{i}^{m}>0} \lambda_{i}^{m} \mathbf{W}_{i}^{m}=\mathbf{A} \mathbf{u}_{i}-\mathbf{A}^{+} \Delta \mathbf{u}_{i},
\end{aligned}
$$

where $\mathbf{A}^{ \pm}$is defined by Eq. (3).

Equation (1) can be discretized as

$$
\mathbf{u}_{i}^{n+1}=\mathbf{u}_{i}^{n}-\frac{\Delta t}{\Delta x}\left(\mathbf{f}_{i+1}-\mathbf{f}_{i}\right)
$$

where the flux

$$
\mathbf{f}_{i}=\mathbf{f}\left(\mathbf{u}_{i}^{0}\right)=\mathbf{A} \mathbf{u}_{i}^{0} .
$$

Using Eqs. (8) and (9) for evaluation of $\mathbf{f}_{i+1}$ and $\mathbf{f}_{i}$, respectively, we obtain

$$
\mathbf{f}_{i}=\mathbf{f}\left(\mathbf{u}_{i}\right)-\mathbf{A}^{+} \Delta \mathbf{u}_{i}, \quad \mathbf{f}_{i+1}=\mathbf{f}\left(\mathbf{u}_{i}\right)+\mathbf{A}^{-} \Delta \mathbf{u}_{i+1} .
$$

Equation (10) can now be rewritten as

$$
\mathbf{u}_{i}^{n+1}=\mathbf{u}_{i}^{n}-\frac{\Delta t}{\Delta x}\left(\mathbf{A}^{-} \Delta \mathbf{u}_{i+1}+\mathbf{A}^{+} \Delta \mathbf{u}_{i}\right) \equiv \mathbf{u}_{i}^{n}+\boldsymbol{\Delta}_{i}^{\text {upwind }} .
$$

This scheme is first-order accurate in space and is called the Godunov scheme (c.f. $[5])$.

A second-order correction to Eq. (12) can be obtained by altering its righthand side as $[\mathbf{3}, \mathbf{4}]$

$$
\mathbf{u}_{i}^{n+1}=\mathbf{u}_{i}^{n}+\Delta_{i}^{u p w i n d}-\frac{\Delta t}{\Delta x}\left(\overline{\mathbf{f}}_{\mathbf{i}+\mathbf{1}}-\overline{\mathbf{f}}_{\mathbf{i}}\right),
$$

where the flux

$$
\overline{\mathbf{f}}_{\mathbf{i}}=\frac{1}{2} \sum_{m=1}^{q}\left|\lambda_{i}^{m}\right|\left(1-\frac{\Delta t}{\Delta x}\left|\lambda_{i}^{m}\right|\right) \overline{\mathbf{W}}_{i}^{m} .
$$

Here $\overline{\mathbf{W}}_{i}^{m}$ is a limited version of the wave $\mathbf{W}_{i}^{m}$, obtained upwindly by comparing $\mathbf{W}_{i}^{m}$ to $\mathbf{W}_{i-1}^{m}$ if $\lambda_{i}^{m}>0$ or to $\mathbf{W}_{i+1}^{m}$ if $\lambda_{i}^{m}<0$. 


\section{Nonlinear system of one-dimensional equations}

\subsection{Flux-difference splitting scheme.}

We consider the following set of nonlinear equations:

$$
\mathbf{u}_{, t}+\mathbf{f}_{, x}=0,
$$

where $\mathbf{f}$ is a nonlinear flux. An upwind scheme that is based on flux-difference splitting decomposes the spatial difference of $\mathbf{u}$ and $\mathbf{f}$ into linear combinations of waves (see Eqs. (7) and (11))

$$
\begin{aligned}
\Delta \mathbf{u} & \equiv \mathbf{u}_{i+1}^{n}-\mathbf{u}_{i}^{n}=\sum_{m} \alpha_{i}^{m} \mathbf{r}_{i}^{m}=\sum_{m} \mathbf{W}_{i}^{m}, \\
\Delta \mathbf{f} & \equiv \mathbf{f}_{i+1}^{n}-\mathbf{f}_{i}^{n}=\sum_{m} \lambda_{i}^{m} \alpha_{i}^{m} \mathbf{r}_{i}^{m},
\end{aligned}
$$

where $\mathbf{r}_{i}^{m}, \alpha_{i}^{m}$, and $\lambda_{i}^{m}$ are respectively the right eigenvector, wave strength, and the eigenvalue of the $m$-th wave component, evaluated at $x_{i}$. The symbol $\Delta$ indicates a difference between the neighboring nodal points.

The system of Eq. (13) can be rewritten in the quasilinear form

$$
\mathbf{u}_{, t}+\mathbf{A}(\mathbf{u}) \mathbf{u}_{, x}=0,
$$

where $\mathbf{A}(\mathbf{u})$ is the $q \times q$ Jacobian matrix which is defined as the derivative of $\mathbf{f}$ with respect to $\mathbf{u}$, so that

$$
d \mathbf{f}=\mathbf{A} d \mathbf{u} .
$$

This differential relation can be replaced by its finite-difference analog, namely,

$$
\Delta \mathbf{f}=\overline{\mathbf{A}} \Delta \mathbf{u} .
$$

Roe in $[\mathbf{6}]$ showed how to construct a mean value $\overline{\mathbf{A}}$ such that the above equation holds exactly for arbitrary pairs of state vectors. For the Roe scheme see Sec. 6.2.

Fluctuation splitting requires that the matrix $\overline{\mathbf{A}}$ be split into its negative and positive parts, $i$. e.

$$
\overline{\mathbf{A}}=\overline{\mathbf{A}}^{-}+\overline{\mathbf{A}}^{+} .
$$

For the linear equations $\mathbf{A}^{ \pm}$are defined by Eq. (3). Then,

$$
\Delta \mathbf{f}=\overline{\mathbf{A}}^{-} \Delta \mathbf{u}+\overline{\mathbf{A}}^{+} \Delta \mathbf{u} .
$$

This expression can be compared with Eq. (11).

The first-order formula for Eq. (13) becomes

$$
\mathbf{u}_{i}^{n+1}=\mathbf{u}_{i}^{n}-\frac{\Delta t}{\Delta x}\left(\mathbf{f}_{i+1 / 2}^{n}-\mathbf{f}_{i-1 / 2}^{n}\right),
$$


where $\mathbf{f}_{i-1 / 2}^{n}$ and $\mathbf{f}_{i+1 / 2}^{n}$ denote the fluxes on the left and right sides of the interfaces. We can find three equivalent formulae for the interface flux:

$$
\begin{aligned}
\mathbf{f}_{i+1 / 2} & =\mathbf{f}_{i}+\overline{\mathbf{A}}^{-} \Delta \mathbf{u} \\
\mathbf{f}_{i+1 / 2} & =\mathbf{f}_{i+1}-\overline{\mathbf{A}}^{+} \Delta \mathbf{u}, \\
\mathbf{f}_{i+1 / 2} & =\frac{1}{2}\left(\mathbf{f}_{i}+\mathbf{f}_{i+1}\right)-|\overline{\mathbf{A}}| \Delta \mathbf{u}
\end{aligned}
$$

where $|\overline{\mathbf{A}}|=\overline{\mathbf{A}}^{+}-\overline{\mathbf{A}}^{-}$.

In practice Eq. (15) is the best choice because of its symmetry condition. The flux $\mathbf{f}_{i+1 / 2}$ is then

$$
\mathbf{f}_{i+1 / 2}=\frac{1}{2}\left(\mathbf{f}_{i}+\mathbf{f}_{i+1}-\sum_{m}\left|\lambda_{i}^{m}\right| \alpha_{i}^{m} \mathbf{r}_{i}^{m}\right) .
$$

As the spectral decomposition of the matrix $|\overline{\mathbf{A}}|$ can sometime be cumbersome, $[\mathbf{7}]$ developed the so-called polynomial upwind scheme in which $|\overline{\mathbf{A}}|$ was replaced by $P_{k}(|\overline{\mathbf{A}}|)$, where $P_{k}$ is a polynomial of degree $k$.

\subsection{Euler equations.}

If we introduce the vector $\mathbf{u}(x, t)$ such that

$$
\mathbf{u}(x, t)=\left(\begin{array}{c}
u_{1} \\
u_{2} \\
u_{3}
\end{array}\right)=\left(\begin{array}{c}
\varrho(x, t) \\
\varrho v(x, t) \\
E(x, t)
\end{array}\right)
$$

and the flux,

$$
\mathbf{f}(\mathbf{u})=\left(\begin{array}{c}
\varrho v \\
\varrho v^{2}+p \\
v(E+p)
\end{array}\right)=\left(\begin{array}{c}
u_{2} \\
\frac{1}{2}(3-\gamma) \frac{u_{2}^{2}}{u_{1}}+(\gamma-1) u_{3} \\
\frac{u_{2}}{u_{1}}\left(\gamma u_{3}-\frac{\gamma-1}{2} \frac{u_{2}^{2}}{u_{1}}\right)
\end{array}\right),
$$

system of hydrodynamic equations can be written in the form of Eq. (14). Here $\varrho$ is mas density, $v$ velocity and $E$ a total energy density.

The Jacobian matrix $\mathbf{A}=\mathbf{f}_{, \mathbf{u}}$ is given as

$$
\mathbf{A}=\left(\begin{array}{ccc}
0 & 1 & 0 \\
-\frac{1}{2}(3-\gamma) \frac{u_{2}^{2}}{u_{1}^{2}} & (3-\gamma) \frac{u_{2}}{u_{1}} & \gamma-1 \\
-\gamma \frac{u_{2} u_{3}}{u_{1}^{2}}+(\gamma-1) \frac{u_{2}^{3}}{u_{1}^{3}} & \gamma \frac{u_{3}}{u_{1}}-\frac{3}{2}(\gamma-1) \frac{u_{2}^{2}}{u_{1}^{2}} & \gamma \frac{u_{2}}{u_{1}}
\end{array}\right)
$$

The eigenvalues of this matrix are:

$$
\lambda^{1}(\mathbf{u})=\frac{u_{2}}{u_{1}}-c_{s}, \quad \lambda^{2}(\mathbf{u})=\frac{u_{2}}{u_{1}}, \quad \lambda^{3}(\mathbf{u})=\frac{u_{2}}{u_{1}}+c_{s},
$$


where

$$
c_{s}=\sqrt{\frac{\gamma p}{\varrho}}
$$

is the sound speed. These eigenvalues are associated with the fact that the information from any point in the flow propagates according to the equations

$$
\frac{d x}{d t}=v
$$

and

$$
\frac{d x}{d t}=v \pm c_{s} .
$$

Equation (17) defines a trajectory $T_{0}$, along which the entropy $s$ is constant and which follows a particle path. Variations in the entropy are convected according to the equation

$$
s_{, t}+v s_{, x}=0 .
$$

Equation (18) defines the Riemann invariants

$$
R_{ \pm}=v \pm \int \frac{\gamma d p}{\varrho c_{s}},
$$

which are constant along the trajectories $T_{ \pm}$of Eq. (18). These trajectories follow forward and backward sound waves in the frame moving with the speed $v$.

For the Euler equations, we have the following diagonalizable equation:

$$
\mathbf{R}^{-1} \mathbf{A R}=\mathbf{\Lambda},
$$

with

$$
\mathbf{R}=\left(\mathbf{r}^{1}, \mathbf{r}^{2}, \mathbf{r}^{3}\right)=\left(\begin{array}{rcr}
1 & 1 & 1 \\
v-c_{s} & v & v+c_{s} \\
H-v c_{s} & \frac{v^{2}}{2} & H+v c_{s}
\end{array}\right)
$$

where:

$$
H=\frac{E+p}{\varrho}
$$

is the enthalpy density and $E$ is the total energy density. The matrix $\boldsymbol{\Lambda}$ is

$$
\boldsymbol{\Lambda}=\left(\begin{array}{rlr}
\lambda^{1} & 0 & 0 \\
0 & \lambda^{2} & 0 \\
0 & 0 & \lambda^{3}
\end{array}\right)=\left(\begin{array}{rrr}
v-c_{s} & 0 & 0 \\
0 & v & 0 \\
0 & 0 & v+c_{s}
\end{array}\right)
$$

This equation proves that the Euler equations are hyperbolic. From this equation it follows that infinitesimal changes propagate along characteristics with speeds $v-c_{s}, v, v+c_{s}$. Note that at the points where $c_{s}=0$ all eigenvalues coincide and the system is not strictly hyperbolic there. 
It is noteworthy that the right eigenvector

$$
\mathbf{r}^{2}(\mathbf{u})=\left(\begin{array}{c}
1 \\
\frac{u_{2}}{u_{1}} \\
\frac{u_{2}^{2}}{2 u_{1}^{2}}
\end{array}\right),
$$

which corresponds to $\lambda^{2}(\mathbf{u})$, is linearly degenerate. Since

$$
\lambda^{2}, \mathbf{u}=\left(-\frac{u_{2}}{u_{1}^{2}}, \frac{1}{u_{1}}, 0\right)
$$

we find that $\lambda^{2}{ }_{, \mathbf{u}} \cdot \mathbf{r}^{2}=0$. So, with $\mathbf{r}^{2}$ neither shocks nor rarefaction waves are associated but contact discontinuities across which there is a jump in the mass density. However, the gas pressure and flow velocity are smooth. The other eigenvalues, $\mathbf{r}^{1}$ and $\mathbf{r}^{3}$, might be either shocks or rarefaction waves, depending on $\mathbf{u}_{l}$ and $\mathbf{u}_{r}$. For the shock wave all state variables are discontinuous. For the rarefaction wave all state variables are continuous.

While numerically solving the Euler equations, it is important to discuss the shock tube problem which takes place at every interface of two neighbour cells.

\section{The shock tube problem}

The shock tube problem can be described as follows. Imagine, there is a thin tube filled with gas that is initially divided by a membrane into two different states. The gas has a higher density and pressure in one half of the tube than in the other half and the gas is motionless. At $t=0$, the membrane is rapidly removed and the gas is allowed to flow, involving three distinct waves: a contact discontinuity (c) in the middle and a shock (s) or a rarefaction wave (r) at the left and the right sides, respectively. For the contact discontinuity the mass density is discontinuous but the pressure and velocity are continuous. The rarefaction wave propagates in the opposite direction to the shock, with the mass density decreasing as the wave passes through. The fluid is accelerated abruptly across the shock wave and smoothly through the rarefaction wave [3]. Four wave patterns are possible: scs, scr, rcs, rcr. The fith pattern, which consists of a vacuum between two central contact discontinuities that are surrounded by two rarefaction waves, is theoretically possible but it can never be realized in practice.

A more complex shock tube problem results for real gases. For instance, for the van der Waals gas a mixed rarefaction wave can arise [8]. In this wave the rarefaction fan is connected with a rarefaction shock. A classical compressive shock and a contact discontinuity propagate toward the low pressure region.

A particular problem corresponding to $\varrho_{l}=p_{l}=3$ and $\varrho_{r}=p_{r}=1$ is called the Sod problem [9]. As the pressure on the left, $p_{l}$, is higher than the right 
one a rightwardly propagating shock wave results. The contact discontinuity which is easily seen on the mass density profiles propagates rightwardly and a rarefaction wave moves leftward.

\section{Rankine-Hugoniot jump condition}

A discontinuity in a solution of Eq. (13) propagates with the speed $c$ which depends on the jump in the solution $\mathbf{u}(x, t)$ across the discontinuity. Suppose a discontinuity moves from left to right. At time $t=t_{1}$ the discontinuity is at the spatial position $x=x_{1}$ and at $t=t_{2}$ at the point $x=x_{2}$, where $x_{1}<x_{2}$ and $t_{1}<t_{2}$. Let the values of $\mathbf{u}$ be given as $\mathbf{u}_{l}$ on the left hand side of the discontinuity and as $\mathbf{u}_{r}$ on the right hand side of it. The system of nonlinear conservation laws of Eq. (13) can be integrated to yield

$$
\mathbf{u}_{l}\left(x_{2}-x_{1}\right)=\mathbf{u}_{r}\left(x_{2}-x_{1}\right)+\mathbf{f}\left(\mathbf{u}_{l}\right)\left(t_{2}-t_{1}\right)-\mathbf{f}\left(\mathbf{u}_{r}\right)\left(t_{2}-t_{1}\right) .
$$

In the limit $x_{2} \rightarrow x_{1}$ and $t_{2} \rightarrow t_{1}$ with

$$
c=\frac{x_{2}-x_{1}}{t_{2}-t_{1}}
$$

we have

$$
c\left(\mathbf{u}_{r}-\mathbf{u}_{l}\right)=\mathbf{f}\left(\mathbf{u}_{r}\right)-\mathbf{f}\left(\mathbf{u}_{l}\right) .
$$

This relation between the shock speed $c$ and the states $\mathbf{u}_{l}$ and $\mathbf{u}_{r}$ is called the Rankine-Hugoniot jump condition.

For the case of scalar equations with $u_{l}$ and $u_{r}$ we get

$$
c=\frac{f\left(u_{r}\right)-f\left(u_{l}\right)}{u_{r}-u_{l}} .
$$

This equation suggests that any jump is allowed, provided the speed $c$ is related via the above formula.

For the case of a system of equations, we define vectors $[\mathbf{u}] \equiv \mathbf{u}_{r}-\mathbf{u}_{l}$ and $[\mathbf{f}] \equiv \mathbf{f}\left(\mathbf{u}_{r}\right)-\mathbf{f}\left(\mathbf{u}_{l}\right)$ while $c$ is a scalar. Now, only certain jumps $\mathbf{u}_{r}-\mathbf{u}_{l}$ are allowed, namely those for which vectors $[\mathbf{u}]$ and $[\mathbf{f}]$ are parallel to each other.

For a system of linear equations with $\mathbf{f}(\mathbf{u})=\mathbf{A u}$, Eq. (19) leads to

$$
c[\mathbf{u}]=\mathbf{A}[\mathbf{u}] .
$$

As a consequence of that $[\mathbf{u}]$ and $c$ are an eigenvector and the associated eigenvalue of the matrix $\mathbf{A}$, respectively. 


\section{The Riemann problem for the Euler equations}

In the Riemann problem, an imaginary membrane, which separates two cells at different states is ruptured, and shock, contact discontinuity, and rarefaction waves are emitted when these two states interact. In other words the Riemann solver is based on the idea that two adjacent arbitrary states will evolve into a set of left- and right-going shocks and rarefactions. With certain assumptions on the flux function $\mathbf{f}(\mathbf{u})$, it is always possible, in principle, to solve the Riemann problem if the states $\mathbf{u}_{l}$ and $\mathbf{u}_{r}$ are sufficiently close to each other. The solution consists of waves travelling with finite velocities. These waves may either be discontinuous shock waves or smooth rarefaction waves. The procedure for constructing the solution of a Riemann problem is called a Riemann solver. The most popular Riemann solver is due to Roe [6].

For the Euler equations, we are looking for the states $\mathbf{u}_{l}^{*}$ and $\mathbf{u}_{r}^{*}$ and speeds $c_{1}<c_{2}<c_{3}$ such that the Rankine-Hugoniot condition of Eq. (19) is satisfied

$$
\begin{aligned}
c_{1}\left(\mathbf{u}_{l}^{*}-\mathbf{u}_{l}\right) & =\mathbf{f}\left(\mathbf{u}_{l}^{*}\right)-\mathbf{f}\left(\mathbf{u}_{l}\right), \\
c_{2}\left(\mathbf{u}_{r}^{*}-\mathbf{u}_{l}^{*}\right) & =\mathbf{f}\left(\mathbf{u}_{r}^{*}\right)-\mathbf{f}\left(\mathbf{u}_{l}^{*}\right), \\
c_{3}\left(\mathbf{u}_{r}-\mathbf{u}_{r}^{*}\right) & =\mathbf{f}\left(\mathbf{u}_{r}\right)-\mathbf{f}\left(\mathbf{u}_{r}^{*}\right) .
\end{aligned}
$$

It can be shown that $c_{2}=v\left(\mathbf{u}_{l}^{*}\right)=v\left(\mathbf{u}_{r}^{*}\right)$, where $v$ is the flow speed [2]. This means that the second discontinuity propagates with the flow speed $\lambda^{2}=v$. Moreover, as $p\left(\mathbf{u}_{l}^{*}\right)=p\left(\mathbf{u}_{r}^{*}\right)$ the pressure is continuous across this discontinuity which is known as contact discontinuity. We remind that across a contact discontinuity mass density, energy, and entropy are discontinuous, but pressure and velocity are continuous. Across shocks all dependent variables change discontinuously. Shocks propagate with a velocity which is uniquely determined through the Rankine-Hugoniot relations of Eq. (19). The first discontinuity would be a shock if the entropy condition is fulfilled

$$
\left(v-c_{s}\right)\left(\mathbf{u}_{l}\right)>c_{1}>\left(v-c_{s}\right)\left(\mathbf{u}_{l}^{*}\right) .
$$

Otherwise, this jump would be a rarefaction wave. Analogously, the third discontinuity is a shock if

$$
\left(v+c_{s}\right)\left(\mathbf{u}_{r}^{*}\right)>c_{3}>\left(v+c_{s}\right)\left(\mathbf{u}_{r}\right) .
$$

A solution of the Riemann problem is shown schematically in the $x$ - and $t$-coordinates in Fig. 1.

The Riemann problem can be solved exactly with the use of Rankine-Hugoniot relations across shocks and the isentropic characteristic equations across rarefaction waves $[\mathbf{1 0}]$. Such procedure leads to a nonlinear algebraic equation (for a flow variable) which can be solved by a Newton iterative method. As 


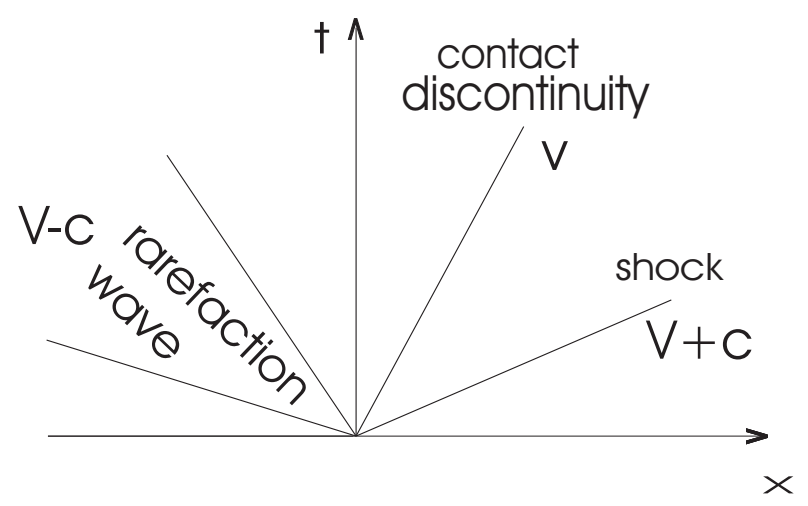

Fig. 1. Wave structure of the Riemann problem for the one-dimensional Euler equations

a consequence of that it is clearly a computationally expensive procedure and therefore different methods are required. These methods are described in the following part of the monograph.

\subsection{The HLL Riemann solver.}

A simple approximate Riemann solver was developed by Harten, Lax, and van Leer in [11]. In this solver (hearafter called HLL) the solution is approximated by two waves which propagate with their speeds $c^{-}$and $c^{+}$such that they correspond to the minimum and maximum characteristic speeds of the system [12]. The wave strengths in the HLL solver are:

$$
\begin{aligned}
& \mathbf{W}^{1}=\mathbf{u}_{*}-\mathbf{u}_{l}, \\
& \mathbf{W}^{2}=\mathbf{u}_{r}-\mathbf{u}_{*},
\end{aligned}
$$

with the middle state $\mathbf{u}_{*}$ that is chosen to preserve conservation

$$
\left(c^{+}-c^{-}\right) \mathbf{u}_{*}=c^{+} \mathbf{u}_{r}-c^{-} \mathbf{u}_{l}-\left(\mathbf{f}\left(\mathbf{u}_{r}\right)-\mathbf{f}\left(\mathbf{u}_{l}\right)\right) .
$$

Hence the intermediate state is obtained as

$$
\mathbf{u}_{*}=\frac{1}{c^{+}-c^{-}}\left[c^{+} \mathbf{u}_{r}-c^{-} \mathbf{u}_{l}-\left(\mathbf{f}\left(\mathbf{u}_{r}\right)-\mathbf{f}\left(\mathbf{u}_{l}\right)\right)\right] .
$$

As the Euler equations evolve three distinctive waves, with its speeds given by Eq. (16), obviously the HLL Riemann solver suffers a drawback and a more appropriate solver is required.

\subsection{The Roe approximate Riemann solver.}

We consider a one-dimensional Riemann problem at the cell edge for the system, given by Eq. (13). On the left side of the edge, there is the state $\mathbf{u}_{l}$, 
on the right side $\mathbf{u}_{r}$. The solution of the Riemann problem for a nonlinear hyperbolic system like the Euler equations in general needs iterative methods which are not very efficient. For small jumps at the interfaces, it is sufficient to use an approximate Riemann solver which is based on a local replacement of the nonlinear equations by a linear hyperbolic system. To appropriately solve the Riemann problem, we can use the following linearized equation:

$$
\mathbf{u}_{, t}+\mathbf{A}(\overline{\mathbf{u}}) \mathbf{u}_{, x}=\mathbf{u}_{, t}+\overline{\mathbf{A}} \mathbf{u}_{, x}=0 .
$$

This idea is used in the Roe scheme $[\mathbf{6}]$ the keystone of which is the introduction of an average Jacobian $\overline{\mathbf{A}}$, which approximates the Jacobian $\mathbf{A}=\mathbf{f}_{, \mathbf{u}}$, associated with the one-dimensional hyperbolic system of conservation laws of Eq. (13). The average Jacobian (called also the Roe matrix) is such that for any given left and right pair of states $\left(\mathbf{u}_{l}, \mathbf{u}_{r}\right)$ the so-called Property $\mathrm{U}$ is satisfied:

(i) $\overline{\mathbf{A}}$ is a linear mapping from the vector space $\mathbf{u}$ to the vector space $\mathbf{f}$;

(ii) $\overline{\mathbf{A}}\left(\mathbf{u}_{l}, \mathbf{u}_{r}\right) \rightarrow \mathbf{f}_{, \mathbf{u}}$ as $\mathbf{u}_{l}$ and $\mathbf{u}_{r} \rightarrow \mathbf{u}$;

(iii) $\overline{\mathbf{A}}\left(\mathbf{u}_{l}, \mathbf{u}_{r}\right)$ has real eigenvalues and a complete set of linearly independent eigenvectors;

(iv) $\overline{\mathbf{A}}\left(\mathbf{u}_{r}-\mathbf{u}_{l}\right)=\mathbf{f}_{r}-\mathbf{f}_{l}$ for any $\mathbf{u}_{l}$ and $\mathbf{u}_{r}$.

In the original Roe scheme, the average state $\overline{\mathbf{u}}$ used to linearize the problem is not $\left(\mathbf{u}_{l}+\mathbf{u}_{r}\right) / 2$. Instead it is taken so that the property (iv) is satisfied. In the case of the Euler or MHD equations, the average mass density is given by

$$
\bar{\varrho}=\sqrt{\varrho_{l} \varrho_{r}}
$$

and the rest of flow variables $(\mathbf{v}, E)$, which are denoted here by $\phi$, are averaged as follows $(e . g .,[\mathbf{1 3}])$ :

$$
\bar{\phi}=\frac{\sqrt{\varrho_{l}} \phi_{l}+\sqrt{\varrho_{r}} \phi_{r}}{\sqrt{\varrho_{l}}+\sqrt{\varrho_{r}}} .
$$

Once all the averaged variables are obtained, the linearized Riemann problem, expressed by Eq. (6.2) is considered at each interface. The exact solution of this approximate problem can be expressed in terms of right eigenvector $\mathbf{r}^{m}$ of $\overline{\mathbf{A}}$ as

$$
\Delta \mathbf{u} \equiv \mathbf{u}_{r}-\mathbf{u}_{l}=\sum_{m=1}^{q} \alpha^{m} \mathbf{r}^{m} .
$$

Here, $q$ corresponds to the number of eigenvectors. The coefficients $\alpha^{m}$ may be determined by multiplying the above equation by each left eigenvector $\mathbf{l}^{j}$. Noting that

$$
\mathbf{l}^{j} \mathbf{r}^{m}=\delta_{j m},
$$

the respective $\alpha^{m}$ is defined by

$$
\alpha^{m}=\mathbf{l}^{m} \Delta \mathbf{u} .
$$


In the above equations the eigenvalues of $\overline{\mathbf{A}}$ are the wavespeeds, the right eigenvectors define the paths taken in phase-space by simple waves. The left eigenvectors define the characteristic equations.

According to property (iv), with the use of Eq. (6.2) we get the vector flux increment expressed as a product of $\Delta \mathbf{u}$ and the corresponding eigenvalues $\lambda^{m}$, viz.

$$
\Delta \mathbf{f}=\mathbf{f}_{r}-\mathbf{f}_{l}=\sum_{m=1}^{q} \alpha^{m} \lambda^{m} \mathbf{r}^{m} .
$$

\subsection{A relaxation Riemann solver.}

In the relaxation scheme of Jin and Xin in [14] Eq. (13) is replaced by a system of coupled equations

$$
\begin{aligned}
\mathbf{u}_{, t}+\mathbf{v}_{, x} & =0 \\
\mathbf{v}_{, t}+\mathbf{B}^{2} \mathbf{u}_{, x} & =\frac{1}{\tau}(\mathbf{f}(\mathbf{u})-\mathbf{v}),
\end{aligned}
$$

where $\mathbf{u}, \mathbf{v} \in R^{q}$ and $\mathbf{B}^{2} \in R^{q \times q}$ is a positive definite matrix. In the original scheme this matrix was chosen to be a diagonal matrix with positive diagonal elements [14]. The relaxation time is denoted by $\tau>0$.

As $\tau \rightarrow 0$ from Eq. (22) we get

$$
\mathbf{v} \rightarrow \mathbf{f}(\mathbf{u})
$$

if a subcharacteristic condition is satisfied

$$
|\lambda| \leq b_{\max }
$$

where $b_{\max }=\max _{m}\left\{b^{m}\right\}$ is the spectral radius of $\mathbf{B}$ with positive eigenvalues of $\mathbf{B}$ such that

$$
b^{m}>0, \quad m=1,2, \cdots, q .
$$

In Eq. (6.3) $\lambda$ is an eigenvalue of the Jacobian matrix $\mathbf{f}_{, \mathbf{u}}$.

Equations (21) and (22) can be advanced with the use of the fractional step method. First the following equations are advanced over that time step $\Delta t$ :

$$
\begin{aligned}
\mathbf{u}_{, t}+\mathbf{v}_{, x} & =0, \\
\mathbf{v}_{, t}+\mathbf{B}^{2} \mathbf{u}_{, x} & =0 .
\end{aligned}
$$

This step leads to $\mathbf{u}^{*}$ and $\mathbf{v}^{*}$ which are updated to $\mathbf{u}^{n+1}$ and $\mathbf{v}^{n+1}$ by solving the equations

$$
\begin{aligned}
\mathbf{u}_{, t} & =0 \\
\mathbf{v}_{, t} & =\frac{1}{\tau}(\mathbf{f}(\mathbf{u})-\mathbf{v})
\end{aligned}
$$


From Eq. (25) we have

$$
\mathbf{u}^{n+1}=\mathbf{u}^{*} .
$$

Equation (26) can be treated implicitly to obtain

$$
\mathbf{v}^{n+1}=\mathbf{f}\left(\mathbf{u}^{n+1}\right)+e^{-\Delta t / \tau}\left[\mathbf{v}^{*}-\mathbf{f}\left(\mathbf{u}^{n+1}\right)\right] .
$$

In the limit of $\tau \rightarrow 0$ this expression simplifies to

$$
\mathbf{v}^{n+1}=\mathbf{f}\left(\mathbf{u}^{n+1}\right) .
$$

In summary, the relaxation scheme consists of solving Eq. (24) and using Eq. (6.3). An approximate Riemann solver is defined then as follows. Given values $\mathbf{u}_{l}$ and $\mathbf{u}_{r}$ we compute $\mathbf{v}_{l}=\mathbf{f}\left(\mathbf{u}_{l}\right)$ and $\mathbf{v}_{r}=\mathbf{f}\left(\mathbf{u}_{r}\right)$ and then solve the Riemann problem for Eq. (21) with the data [15]

$$
\left\{\begin{array} { l } 
{ \mathbf { u } _ { l } , } \\
{ \mathbf { f } ( \mathbf { u } _ { l } ) , }
\end{array} \quad \left\{\begin{array}{l}
\mathbf{u}_{r}, \\
\mathbf{f}\left(\mathbf{u}_{r}\right) .
\end{array}\right.\right.
$$

\subsection{Extension of the Roe scheme for a general equation of state.}

The Roe scheme was originally devised for a perfect gas. This scheme is valid for fluid of a constant ratio of specific heats, $\gamma$. One way to extend this scheme for a variable $\gamma$ is to adopt a mean value of $\gamma$. The other options are based on application of some sort of averaging $[\mathbf{1 6}, \mathbf{1 7}, \mathbf{1 8}, \mathbf{8}]$. Here, we describe briefly the method which was developed by Hanawa, Nakajima, and Nobuta [19].

It is useful to introduce the specific total enthalpy $H$ and the specific enthalpy $h$ as

$$
H=E+\frac{p}{\varrho}=h+\frac{v^{2}}{2}
$$

and assume that thermodynamic variables depend on the mass density $\varrho$ and the specific internal energy $e$. The Jacobian matrix possesses the right eigenvectors

$$
\begin{aligned}
\mathbf{r}^{1}=\frac{\bar{\varrho}}{2 \bar{c}}\left(\begin{array}{c}
1 \\
\bar{v}+\bar{c} \\
\bar{H}+\bar{v} \bar{c},
\end{array}\right), \quad \mathbf{r}^{2}=\left(\begin{array}{c}
1 \\
\bar{v} \\
\frac{1}{2} \bar{v}^{2}+\varepsilon,
\end{array}\right), \\
\mathbf{r}^{3}=\frac{\bar{\varrho}}{2 \bar{c}}\left(\begin{array}{c}
1 \\
\bar{v}-\bar{c} \\
\bar{H}-\bar{v} \bar{c}
\end{array}\right)
\end{aligned}
$$

and the corresponding eigenvalues

$$
\lambda^{1}=\bar{v}+\bar{c}, \quad \lambda^{2}=\bar{v}, \quad \lambda^{3}=\bar{v}-\bar{c},
$$

where $\bar{c}$ is the averaged sound speed such as

$$
\bar{c}^{2}=(\gamma-1)\left(\bar{H}-\frac{1}{2} \bar{v}^{2}\right)
$$


and

$$
\varepsilon=\frac{\varrho_{i+1} E_{i+1}-\varrho_{i} E_{i}-\frac{1}{\gamma-1}\left(p_{i+1}-p_{i}\right)}{\varrho_{i+1}-\varrho_{i}-\frac{1}{\bar{c}^{2}}\left(p_{i+1}-p_{i}\right)} .
$$

The other averaged quantities are

$$
\begin{aligned}
\bar{\varrho} & =\sqrt{\varrho_{i} \varrho_{i+1}} \\
\bar{v} & =\frac{\sqrt{\varrho_{i}} v_{i}+\sqrt{\varrho_{i+1}} v_{i+1}}{\sqrt{\varrho_{i}}+\sqrt{\varrho_{i+1}}}, \\
\bar{H} & =\frac{\sqrt{\varrho_{i}} H_{i}+\sqrt{\varrho_{i+1}} H_{i+1}}{\sqrt{\varrho_{i}}+\sqrt{\varrho_{i+1}}}
\end{aligned}
$$

with

$$
\frac{1}{\gamma-1}=\frac{\sqrt{\varrho_{i}} h_{i}+\sqrt{\varrho_{i+1}} h_{i+1}}{\sqrt{\varrho_{i}} \frac{\partial p}{\partial \varrho_{i}}+\sqrt{\varrho_{i+1}} \frac{\partial p}{\partial \varrho_{i+1}}} .
$$

The derivative $\frac{\partial p}{\partial \varrho}$ is evaluated at the constant entropy.

The eigenvectors $\mathbf{r}^{1}$ and $\mathbf{r}^{3}$ correspond to the sound waves and the eigenvector $\mathbf{r}^{2}$ is associated with the entropy wave. The amplitude of each wave is given as

$$
\begin{aligned}
\alpha^{1} & =v_{i+1}-v_{i}+\frac{p_{i+1}-p_{i}}{\bar{\varrho} \bar{c}}, \\
\alpha^{2} & =\varrho_{i+1}-\varrho_{i}-\frac{p_{i+1}-p_{i}}{\bar{c}^{2}}, \\
\alpha^{3} & =-v_{i+1}+v_{i}+\frac{p_{i+1}-p_{i}}{\bar{\varrho} \bar{c}} .
\end{aligned}
$$

This scheme consists in a natural generalization of the Roe scheme [6]. In the case of the perfect gas law $p=(\gamma-1) \varrho e$ and the scheme reduces to the Roe scheme.

\section{Deficiencies of Godunov-type schemes}

Godunov-type schemes are very robust and give reliable results for a wide range of problems without the need to be retuned. However, even these modern schemes are far from being perfect. There are few instances in which a particular scheme produces inappropriate results $[\mathbf{2 0}, \mathbf{2 1}]$. For instance, most Godunovtype schemes lead to the generation of a long wavelength noise, downstream nearly stationary shock. This noise is not effectively damped by the dissipation of the scheme [20]. In a few cases Roe solvers exhibit nonlinear instability, producing unphysical local features which are called carbuncles [22]. These features become more pronounced for a finer grid. In multi-dimensions a problem occurs if a wave is far from aligned with the grid. Then, a grid-oblique wave may be represented by grid-aligned waves, enhancing numerical dissipation and leading to a loss of resolution. 
Additionally, the Roe method can admit spurious solutions that are triggered by an incorrect treatment of shear waves. A weighted average flux formulation was used by Quirk [23] to devise a shear fix for Roe's method.

\subsection{Entropy fix.}

Another deficiency of a Godunov-type scheme is that while computing rarefaction waves, the scheme can produce nonphysical expansion shocks in the computed flow. In this case the true Riemann solution contains a transonic wave with characteristic speeds that increase from negative to positive values through the rarefaction fan. Then, the eigenvalue of the average Jacobian $\overline{\mathbf{A}}$ is such that $\lambda_{l}<0$ to the left of the wave while $\lambda_{r}>0$ to the right of the wave. It leads to information travelled partly to the left and partly to the right, affecting cell averages on both sides. The Roe solver approximates every wave by a single discontinuity that propagates at a speed given by an eigenvalue $\bar{c}$ of $\overline{\mathbf{A}}$. In the transonic rarefaction case this speed is approximately zero and the proper spreading does not occur. This can lead to numerical approximations with entropy violating discontinuities.

Several ways to fix the problem of preventing the Roe method from admitting expansion shocks exist in literature. For instance, Yee, Warming, and Harten in $[24]$ replaced the values of the numerical viscosity $\mu$ smaller than some tolerance $\varepsilon$ with higher values $\mu^{\prime}$ such that

$$
\mu^{\prime}= \begin{cases}\mu & \text { for }|\mu| \geq \varepsilon \\ \frac{\mu}{2}\left(\frac{2 \mu^{2}}{\varepsilon}+\frac{\varepsilon}{2}\right) & \text { for }|\mu|<\varepsilon .\end{cases}
$$

For typical simulations $\varepsilon$ is set to 0.2 . This modification is only applied to rarefaction waves. Although the dependence on $\varepsilon$ is small and this entropy works well it suffers from a drawback that a tunable parameter $\varepsilon$ was introduced into the scheme and there is little physical justification for its use.

To prevent the expansion shocks, an intermediate state (that simulates the diffusion) between the left and right states is introduced [25]. More precisely, the single wave $\bar{\alpha} \overline{\mathbf{r}}$ (coming from the Roe solver) is replaced by a pair of waves $\alpha_{l} \mathbf{r}$ and $\alpha_{r} \mathbf{r}$, propagating at speeds $\lambda_{l}<0<\lambda_{r}$. These speeds are chosen to approximate the characteristic speeds at each edge of the rarefaction fan. As the total wave strength should remain the same, we require

$$
\alpha_{l}+\alpha_{r}=\bar{\alpha} .
$$

To maintain conservation we also need

$$
\alpha_{l} \lambda_{l}+\alpha_{r} \lambda_{r}=\bar{\alpha} \bar{c} .
$$


From these equations we obtain

$$
\begin{aligned}
\alpha_{l} & =\frac{\bar{\alpha} \lambda_{r}-\bar{c}}{\lambda_{r}-\lambda_{l}}, \\
\alpha_{r} & =\frac{\bar{c}-\bar{\alpha} \lambda_{l}}{\lambda_{r}-\lambda_{l}} .
\end{aligned}
$$

The above procedure is called an entropy fix. This procedure is necessary to obtain physically relevant numerical approximations of the exact solution. In the Roe method $[\mathbf{6}]$ one only needs an entropy fix at sonic points $[\mathbf{2 6}]$. There was some debate if this is the same for the magnetohydrodynamic waves. However, the simplest approach is to apply the entropy fix for the magnetosonic (fast and slow) waves only. The Alfvén and entropy waves are supposed not to need entropy fixes as they are linearly degenerate.

A very popular entropy fix method was developed by LeVeque in [2]. The idea used in the entropy fix is to replace the single jump $\mathbf{u}_{r}-\mathbf{u}_{l}$, propagating at speed $\lambda$ by two jumps propagating at speeds $\lambda_{l}$ and $\lambda_{r}$, with a new state $\mathbf{u}_{*}$ in between $[\mathbf{2 5}]$.

The flux difference can be expressed as follows:

$$
\mathbf{f}\left(\mathbf{u}_{l}\right)-\mathbf{f}\left(\mathbf{u}_{r}\right)=\mathbf{f}\left(\mathbf{u}_{l}\right)-\mathbf{f}\left(\mathbf{u}_{*}\right)+\mathbf{f}\left(\mathbf{u}_{*}\right)-\mathbf{f}\left(\mathbf{u}_{r}\right) .
$$

Using the property (iv) of the Roe solver this formula can be rewritten as

$$
\lambda\left(\mathbf{u}_{l}-\mathbf{u}_{r}\right)=\lambda_{l}\left(\mathbf{u}_{l}-\mathbf{u}_{*}\right)+\lambda_{r}\left(\mathbf{u}_{*}-\mathbf{u}_{r}\right) .
$$

Hence,

and consequently

$$
\mathbf{u}_{*}=\frac{\left(\lambda-\lambda_{l}\right) \mathbf{u}_{l}+\left(\lambda_{r}-\lambda\right) \mathbf{u}_{r}}{\lambda_{r}-\lambda_{l}}
$$

$$
\begin{aligned}
& \mathbf{u}_{*}-\mathbf{u}_{l}=\frac{\lambda_{r}-\lambda}{\lambda_{r}-\lambda_{l}}\left(\mathbf{u}_{r}-\mathbf{u}_{l}\right) \equiv \frac{\lambda_{r}-\lambda}{\lambda_{r}-\lambda_{l}} \alpha^{j} \mathbf{r}^{j} \\
& \mathbf{u}_{r}-\mathbf{u}_{*}=\frac{\lambda-\lambda_{l}}{\lambda_{r}-\lambda_{l}}\left(\mathbf{u}_{r}-\mathbf{u}_{l}\right) \equiv \frac{\lambda-\lambda_{l}}{\lambda_{r}-\lambda_{l}} \alpha^{j} \mathbf{r}^{j} .
\end{aligned}
$$

The flux can now be written twofold $[\mathbf{2}]$

$$
\mathbf{f}\left(\mathbf{u}_{l}, \mathbf{u}_{r}\right)=\mathbf{f}\left(\mathbf{u}_{l}\right)+\sum_{m \neq j} \lambda^{-} \alpha^{m} \mathbf{r}^{m}+\hat{\lambda}_{l}^{j} \alpha^{j} \mathbf{r}^{j}
$$

or

$$
\mathbf{f}\left(\mathbf{u}_{l}, \mathbf{u}_{r}\right)=\mathbf{f}\left(\mathbf{u}_{r}\right)-\sum_{m \neq j} \lambda^{+} \alpha^{m} \mathbf{r}^{m}-\hat{\lambda}_{r}^{j} \alpha^{j} \mathbf{r}^{j},
$$

where we introduced the following notation:

$$
\hat{\lambda}_{l}^{j} \equiv \lambda_{l} \frac{\lambda_{l}-\lambda}{\lambda_{r}-\lambda_{l}}, \quad \hat{\lambda}_{r}^{j} \equiv \lambda_{r} \frac{\lambda-\lambda_{l}}{\lambda_{r}-\lambda_{l}} .
$$


The eigenvalue $\lambda^{ \pm}$is defined by Eq. (4). Equations (27) and (28) are used instead of Eqs. (8) and (9).

Another entropy fix that was based on estimates for the spreading rate of each wave within the approximate Riemann solution was devised by Roe in [27]. Moreover, Osher [28] found a general condition for a scheme to be entropy satisfying when applied to scalar equations. He designed such schemes which were called E-schemes.

The above presented entropy fixes may fail in some circumstances. For instance, it has been found that the above entropy fix failed in the presence of a negative/positive transonic rarefaction of the van der Waals gas [8]. In this case the standard entropy fix of $[\mathbf{2 5}]$ overcome this problem $[\mathbf{8}]$.

\section{Numerical simulations of random waves}

In recent years, the propagation of waves in random media has been the subject of intensive studies in the most diverse branches of physics. For instance, the effect of turbulence in the solar atmosphere on the surface-gravity wave amplitudes and frequencies was discussed by Murawski in $[\mathbf{2 9}, \mathbf{3 0}]$. In another context, $[\mathbf{3 1}]$ showed that a space-dependent random flow shifts the frequencies of acoustic waves and alters their amplitude. Fast magnetosonic waves which are impulsively generated in plasma, having a random mass density, were discussed by Murawski, Nakariakov, and Pelinovsky in [32]. They showed that the localized pulses experience a spatial delay and attenuation due to the spacedependent (step-wise constant) random field. Attenuation of nonlinear waves was considered by Wadati [33] who proved that a soliton, propagating a distance $x$ in a $x$-dependent random medium, has its amplitude decreased as $1 / \sqrt{x}$ and its width increased as $\sqrt{x}$. Lipkens and Blanc-Benon in [34] showed that as a result of turbulence the nonlinear distortion of a pulse is weaker than in the deterministic case.

We consider here a simple case of one-dimensional acoustic waves propagating in a fluid whose density is a random function of time. To provide a consistent and general treatment of wave propagation, we develop a numerical approach for which the deviations of the random density fluctuations from their mean value and the wave amplitude are not necessarily small.

In this section, we present the results of the numerical simulations for onedimensional Euler equations. These simulations are performed with the use of the CLAWPACK code $[\mathbf{3}, \mathbf{4}, \mathbf{3 5}]$, which is a packet of Fortran routines for solving hyperbolic equations. The code utilizes the wave propagation method ([3] and Sec. 2.3).

$$
\varrho_{0}=\text { const }, v_{0}=0, p_{0}=\text { const } .
$$


Here $\varrho_{0}, v_{0}$, and $p_{0}$ are the background mass density, velocity, and pressure, respectively.

\subsection{Numerical results for a random field.}

The random field is seeded through the term $S_{\varrho}$ in the momentum equation such that

$$
S_{\varrho}=\varrho_{r, t},
$$

where:

$$
\begin{array}{r}
\varrho_{r}\left(t_{m}\right)=\Re\left(\sqrt{\frac{2}{N}} \sum_{n=0}^{N-1} \bar{\varrho}\left(\Omega_{n}\right) e^{\left(-2 j \pi m \frac{n}{N}+j \phi_{n}\right)}\right) \equiv \\
\sqrt{2 N} \Re\left(\mathcal{F}^{-1}\left(\bar{\varrho}\left(\Omega_{n}\right) e^{-\phi_{n}}\right)\right) .
\end{array}
$$

Here $N$ denotes number of Fourier modes taken into account, $\phi_{n}$ is a random phase with uniform distribution over the interval $\langle 0,2 \pi)$, chosen by the random number generator ran1 $[\mathbf{3 6}]$. The quantity $\varrho\left(\Omega_{n}\right)$ denotes the mode's amplitude

$$
\bar{\varrho}\left(\Omega_{n}\right)=\sqrt{E\left(\Omega_{n}\right)} .
$$

The spectrum $E(\Omega)$ is sampled uniformly by $N$ values $\Omega_{n}$

$$
\Omega_{n}=\frac{2 \pi n}{N \Delta t}, \quad n=0,1,2, \cdots, N-1,
$$

where:

$$
\Delta t=\frac{t_{N-1}-t_{0}}{N}
$$

In Eq. (29) $t$ is sampled as follows:

$$
t_{m}=t_{0}+m \Delta t, \quad m=0,1,2, \cdots, N-1 .
$$

With the use of the correlation theorem $([\mathbf{3 6}])$ :

$$
\mathcal{F}<\varrho_{r}\left(t_{m_{1}}\right) \varrho_{r}\left(t_{m_{2}}\right)>=\left|\varrho_{r}(k)\right|^{2}
$$

we find that the random field, which is generated in this way, possesses the required spectral properties. Fig. 2 shows typical realization of the random field which was generated by the present method. A similar way of seeding the random field was developed by Juvé et al. in [37].

The periodic boundary conditions are applied at the edges of the simulation region which are typically chosen at $x=0$ and $x \simeq 82 c_{0} l_{t}$. 


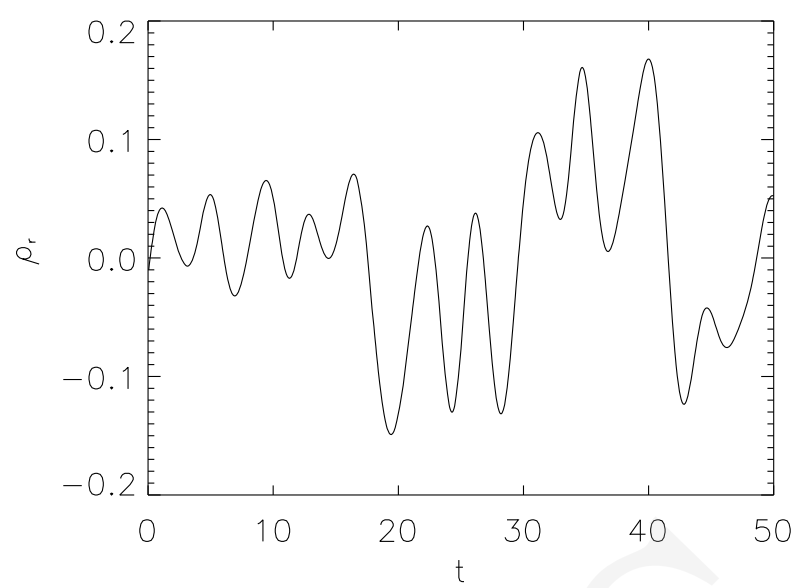

Fig. 2. Random mass density as a function of time $t$ for typical accomplishment of random medium

\section{Conclusions}

In this paper we presented the Godunov-type numerical methods for solving hyperbolic equations. Although this presentation is far from complete the emphasis is on the methods which are the most effective and the best known for the author.

There are several conditions that numerical schemes should satisfy: accuracy and speed of numerical simulations, adequate representation of complex flows and steep profiles, without generation of spurious oscillations as well as robustness. A computer code is described as being robust if it has the virtue of giving reliable results to a wide range of problems without the need to be retuned. Modern numerical schemes such as shock-capturing schemes described in this paper satisfy these conditions.

The existing numerical models demonstrate the feasibility of fluid simulations in obtaining at least qualitative and, to some extent, quantitative features in the magnetized fluid. With continuous improvement in computational methods and computer resources, the usefulness and capability of the numerical approach should continue to develop.

We demonstrated a performance of the CLAWPACK code as an example of a numerical study of a medium with time-dependent random mass density fluctuations.

This work was performed in the frame of the grant from the Polish Ministry of Science for the years 2007-2010. 


\section{References}

[1] Jeffrey A., Taniuti T., Nonlinear waves propagation, Academic Press, New York 1964.

[2] LeVeque R.J., Numerical methods for conservation laws, Birkhäuser Verlag Basel, Berlin 1990.

[3] LeVeque R.J., CLAWPACK User Notes, Applied Mathematics, Univ. of Washington, Washington, Seattle 1997, available from http://www.amath.washington.edu/rjl/clawpack.html

[4] LeVeque R.J., Wave propagation algorithm for multi-dimensional hyperbolic systems, J. Comp. Phys. 131 (1997) 327.

[5] Godunov S.K., A difference method for numerical calculation of discontinuous equations of hydrodynamics, Math. Sb. 47 (1959) 271.

[6] Roe P.L., Approximate Riemann solvers, parameter vectors, and difference methods, J. Comp. Phys 43 (1981) 357.

[7] Degond P., Peyrard P.F., Russo G., Villedieu P., Polynomial upwind schemes for hyperbolic systems, C. R. Acad. Sci. Paris 328 (1999) 479.

[8] Guardone A., Vigevano L., Roe linearization for the van der Waals gas, J. Comp. Phys. 175 (2002) 50 .

[9] Sod G., A survey of finite difference methods for systems of nonlinear hyperbolic conservation laws, J. Comp. Phys., 27 (1978) 1.

[10] Gottlieb J.J., Groth C.P.T., Assessment of Riemann solvers for unsteady one-dimensional inviscid flows of perfect gases, J. Comp. Phys. 78 (1998) 437.

[11] Harten A., Lax P.D., van Leer B., On upstream differencing and. Godunov-type schemes for hyperbolic conservation laws, SIAM Rev. 25 (1983) 35.

[12] Einfeld B., On Godunov-type methods for gas dynamics, SIAM J. Num. Anal. 25 (1988) 294.

[13] Aslan N., Two dimensional solutions of MHD equations with an adapted Roe Method Int. J. Numer. Meth. Fluids 23 (1996) 1211.

[14] Jin S., Xin Z.P., The relaxation schemes for systems of conservation laws in arbitrary space dimensions, Comm. Pure Appl. Math. 48 (1995) 235.

[15] LeVeque R.J., Pelanti M., A class of approximate Riemann solvers and their relation to relaxation schemes, J. Comput. Phys. 172 (2001) 572.

[16] Glaister P., An efficient algorithm for compressible flows with real gases, J. Comp. Phys. 74 (1988) 382.

[17] Toumi I., A weak formulation of Roes approximate Riemann solver, J. Comp. Phys. 102 (1992) 360.

[18] Saurel R., Larini M., Loraud J.C., Exact and approximate Riemann solvers for real gases, J. Comp. Phys. 112 (1994) 126.

[19] Hanawa T., Nakajima Y., Nobuta K., Roe scheme for a general equation of state, Nagoya University 1999, preprint.

[20] Donat R., Marquina A., Capturing shock reactions: an improved fux formula, J. Comp. Phys. 125 (1996) 42.

[21] Einfeld B., Munz C.D., Roe P.L., Sjögreen B., On Godunov-type methods near low densities, J. Comp. Phys. 92 (1991) 273.

[22] Quirk J.J., A contribution to the great Riemann solver debate, Int. J. Numer. Methods Fluids 18 (1994) 555. 
[23] Quirk J.J., An adaptive grid algorithm for computational shock hydrodynamics, PhD thesis, College of Aeronautics 1991.

[24] Yee H.C., Warming R.F., Harten A., Explicit and implicit high-resolution shock-capturing methods, J. Comp. Phys. 57 (1985) 327.

[25] Harten A., Hyman J.M., Self-Adjusting Grid for One-Dimensional Hyperbolic Conservation Laws, J. Comp. Phys. 50 (1983) 235.

[26] Roe P.L., Sonic flux formulae, SIAM J. Sci. Stat. Comput. 13 (1982) 611.

[27] Roe P.L., Finite-volume methods for the compressible Navier-Stokes equations in Lectures in Applied Mathematics, Large-Scale. Computations in Fluid Mechanics 22 (1985). American Mathematical Society: Providence, RI.

[28] Osher S., High resolution schemes using flux limiters for hyperbolic equations, SIAM J. Num. Anal. 21 (1984) 289.

[29] Murawski K., Influence of coherent and random flows on the solar f-mode, Astrophys. J. 537 (2000) 495.

[30] Murawski K., Turbulent f-mode in a stratified solar atmosphere, Astron. Astrophys. 360 (2000) 707.

[31] Murawski K., Influence of a stochastic flow on acoustic waves, Acta Astron. 50 (2000) 269.

[32] Murawski K., Nakariakov V.N., Pelinovsky E.N., Fast magnetoacoustic waves in a randomly structured solar corona, Astron. Astrophys. 366 (2001) 306.

[33] Wadati M., Deformation of solitons in random media, J. Phys. Soc. Japan 59 (1990) 4201.

[34] Lipkens B., Blanc-Benon Ph., Propagation of finite-amplitude sound through stochastic media, C. R. Acad. Sci. Paris 320 (1995) 477.

[35] LeVeque R.J., in Computational methods for astrophysical fluid flow, Steiner O., Gautschy A., (eds.), 27th Saas-Fee Adv. Course Lect. Notes, Springer-Verlag, Les Diablerets 1997.

[36] Press W.H., Flannery B.P., Teukolsky S.F., Vetterling W.T., Numerical recipes, Cambridge Univ. Press, Cambridge 1992.

[37] Juvé D., Blanc-Benon Ph., Wert K., in Theoretical and Computational Acoustics '97, Teng et al. Y.-C., (eds.), World Sc. Pub. Co., Singapore 1999. 